\title{
Instabilities in first order phase transitions*
}

\author{
S BANERJEE \\ Metallurgy Programme, Bhabha Atomic Research Centre, Bombay 400085, India
}

\begin{abstract}
Majority of the metallurgical phase transformations are first-order transitions which occur by the nucleation and growth process near equilibrium conditions. In recent years, homogeneous transformation has been reported in some of these cases at conditions significantly away from those of equilibrium. In this paper some of these transformations will be discussed. In the first part of the presentation the thermodynamic and the mechanistic distinctions between first and higher order phase transformations will be discussed and a comparison made between homogeneous and heterogeneous modes of phase transformations and those of deformation. Based on Landau's free energy vs generalised order parameter plots, an instability temperature is defined for first order phase transformations below which the transformation can occur by a continuous amplification of a concentration or a strain fluctuation. In the second part experimental evidence in support of the continuous mode of transformations in two ordering reactions are presented. These are: (i) a transition from the short range to the long range chemical order in $\mathrm{Ni}_{4} \mathrm{Mo}$ (D1a structure) and (ii) a hybrid displacive-replacive ordering in $\mathrm{Zr}_{2} \mathrm{Al}$ ( $\mathrm{B} 8_{2}$ structure). In order to make the continuous mode operative in these first order transformations (which is possible at a high "supercooling"), radiation in the former and rapid quenching in the latter were employed. In the last part, the martensitic transformation and the shape memory effect is described in terms of Landau's plots and the mechanical and thermodynamical consequences of the model are discussed.
\end{abstract}

\section{Discussion}

$M$ Youssuff: What is the criterion by which we can distinguish whether the development of long-range order is by nucleation and growth or by a continuous mechanism?

S Banerjee: The only criterion is whether we are below $T_{0}$ or not. Also, not all ordering can be described in terms of compositional modulations.

V K Wadhawan: What is order parameter in $\mathrm{Ni}_{4} \mathrm{Mo}$ ?

Banerjee: The population density of Mo atoms on a given lattice site.

V C Sahni: Is there any example where there is a formation volume and the transformation is homogeneous?

Banerjee: It is possible that the volume change is very small like in $\mathrm{Ni}_{\mathbf{4}} \mathbf{M o}$.

Sahni: How is the expansion of free energy in terms of the order parameter justified in a first order phase transition (since the OPM is discontinuous)?

Banerjee: It cannot be justified.

* Only a summary is presented. 